\title{
Energy-transfer in porphyrin- functionalized graphene
}

\author{
Ermin Malic ${ }^{*}, 1,2$, Heiko Appel ${ }^{2}$, Oliver T. Hofmann², and Angel Rubio ${ }^{2,3}$ \\ ${ }^{1}$ Institut für Theoretische Physik, Technische Universitt Berlin, Hardenbergstr. 3610623 Berlin, \\ ${ }^{2}$ Fritz-Haber-Institut der Max-Planck-Gesellschaft, Faradayweg 4-6, D-14195 Berlin, Germany, \\ ${ }^{3}$ Nano-Bio Spectroscopy group and ETSF Scientific Development Centre, Universidad del Pais Vasco, Avenida de Tolosa 72, E-20018 \\ Donostia, Spain
}

Received XXXX, revised XXXX, accepted XXXX

Published online XXXX

Key words: functionalization, graphene, porphyrin, energy transfer, Frster coupling

*Corresponding author: e-mail: ermin.malic@tu-berlin.de, angel.rubio@ehu.es

We present a theoretical study on the moleculesubstrate interaction within the porphyrin-functionalized graphene. Recent experiments on porphyrinfunctionalized carbon nanotubes have revealed an extremely efficient energy transfer from the adsorbed molecules to the carbon substrate. To investigate the energy transfer mechanism, we have characterized the hybrid structure within the density functional theory including the calculation of the molecular transition dipole moment, which allows us to determine the Förster coupling rate. We find a strongly pronounced Förster-induced energy transfer in the range of $\mathrm{fs}^{-1}$ confirming the experimental observations.

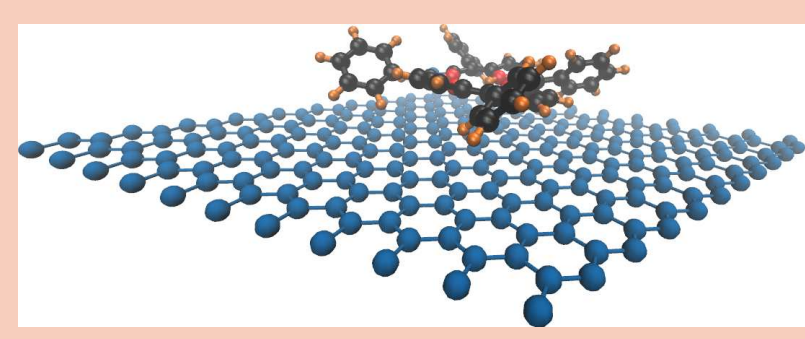

Side view on the graphene layer non-covalently functionalized with a porphyrin molecule.

Copyright line will be provided by the publisher

Functionalization of carbon nanostructures, such as graphene and carbon nanotubes, is a rapidly developing research field [1-25]. The goal is the design of tailored hybrid nanostructures characterized by remarkable properties of the carbon substrate [26-31] combined with the strong light absorption of adsorbed molecules [32]. This results in a promising application potential for high-efficiency photodetectors, biomedical sensors, and photovoltaic devices [24,33-35]. To preserve to a large extent the intrinsic properties of the carbon substrate, non-covalent functionalization based on $\pi-\pi$ stacking is applied [5].

First experimental studies on carbon-based hybrid nanostructures have demonstrated an extremely efficient energy transfer $[11,12,24,32]$. In particular, hybrid structures consisting of carbon nanotubes non-covalently functionalized with porphyrin molecules show a total quench- ing of the porphyrin fluorescence, while the photoluminescence of the nanotubes is strongly enhanced, when the excitation is tuned into resonance with molecular transitions [36-38]. In transient absorption spectroscopy experiments, an ultrafast population build-up was observed in carbon nanotubes after optical excitation of the porphyrin molecules suggesting that the energy transfer occurs on a timescale shorter than 100 fs [39]. The underlying energy transfer mechanism is expected to be the Förster coupling [40] inducing a direct non-radiative transfer of energy from the optically excited molecule to the substrate. The competing Dexter energy transfer mechanism [41] is expected to be small due to its sensitive dependence on the overlap of the involved strongly localized orbital functions of the carbon substrate and the adsorbed molecules [42]. 
(a)

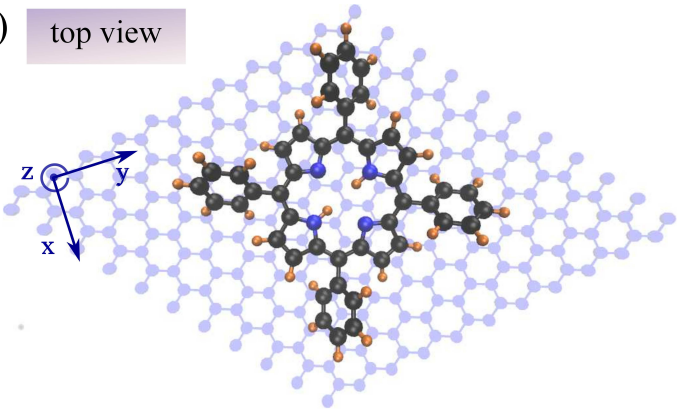

(b)

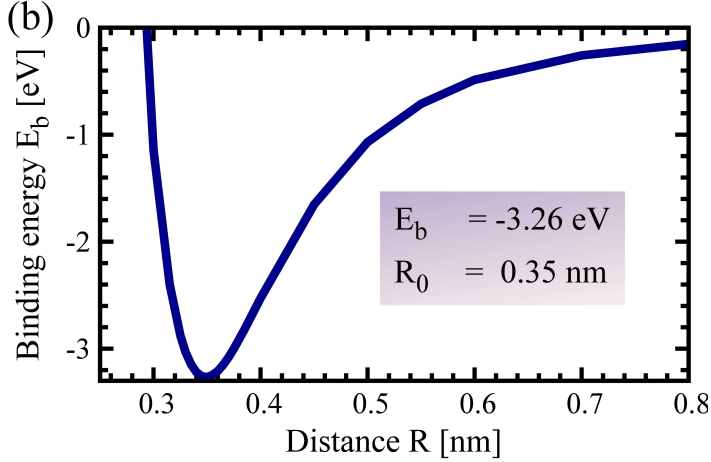

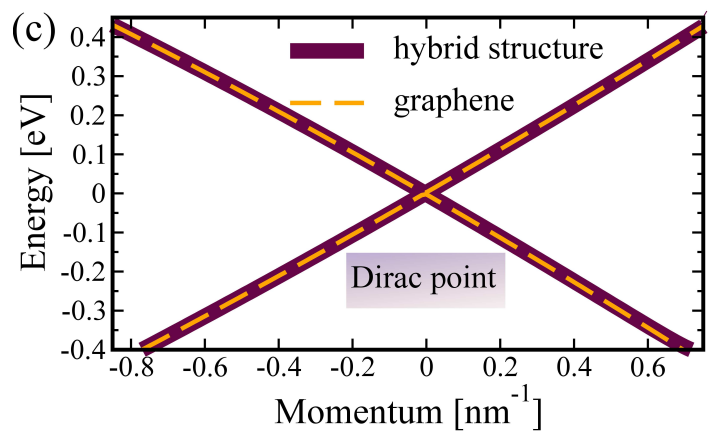

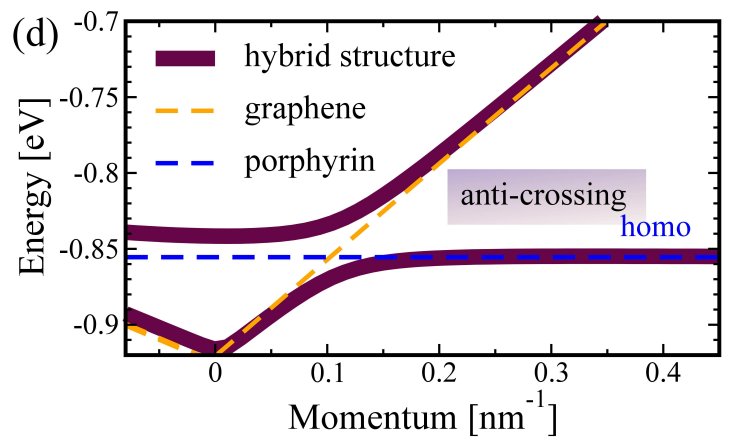

Figure 1 Characterization of the investigated hybrid nanostructure consisting of graphene non-covalently functionalized with base-free tetraphenyl porphyrin (TPP) molecules. (a) Top view on the structure that has been geometrically fully relaxed within the FHI-aims code including the van der Waals interaction $[43,44]$. (b) The total binding energy $E_{b}$ as a function of the molecule-substrate distance $R$. (c) Electronic bandstructure of the hybrid nanostructure at equilibrium graphene-porphyrin distance $R_{0}$ exhibits no changes around the Dirac point with respect to the bandstructure of pristine graphene. (d) Avoided crossings can be observed, when the molecular energy levels cross the graphene bands. The almost entirely unchanged bandstructure and the relatively small binding energy of $42 \mathrm{meV}$ per atom in the porphyrin molecule (78 atoms) characterize the non-covalent functionalization that is less invasive than the covalent adsorption of molecules.

In this article, we present a theoretical study on the Förster energy transfer mechanisms in the graphene functionalized with base-free tetraphenyl porphyrin (TPP). First, we perform a characterization of the investigated hybrid nanostructure based on calculations within the density functional theory. The study is performed within the FHI-aims code package, which is an all-electron fullpotential electronic structure code including numerical atom-centered orbitals allowing the investigation of structures with hundreds of atoms [43]. In the next step, we exploit the gained insights for the evaluation of the Förster energy transfer rate within the Fermi's golden rule [45].

Figure 1(a) illustrates the top view on the investigated porphyrin-functionalized graphene. The hybrid structure is fully relaxed using the Broyden-Fletcher-Goldfarb-Shanno method minimizing all force components to values smaller than $10^{-3} \mathrm{eV} / \AA$. For graphene, we choose a supercell covering $11 x 11$ unit cells corresponding to 242 carbon atoms with a lattice constant of $1.42 \AA$. The investigated situation corresponds to a moderate functionalization degree with a negligible molecule-molecule interaction. The calculations are performed within the PBE exchange-correlation functional [46] including the recently implemented Van der Waals correction [44] to account for the long-rang van der Waals interaction. Calculations of the geometrically relaxed structure reveal an optimal substrate-molecule distance of $R_{0}=0.35 \mathrm{~nm}$ corresponding to the Van der Waals diameter of carbon atoms, cf. Fig. 1(b). The distance is determined with respect to the central part of the molecule. The binding energy of $E_{b}=3.26 \mathrm{eV}$ corresponds to $\hat{E}_{b}=42 \mathrm{meV}$ per atom in the porphyrin molecule (78 atoms). Furthermore, as expected in the case of non-covalent functionalization, we find only small deviations in the electronic band structure compared to the electronic properties of the pristine graphene, cf. Figs. 1 (c) and (d). The molecules adsorbed via $\pi-\pi$ stacking do not change the unique linear and gapless band structure of graphene around the Dirac point. Their impact is limited to regions where the molecular energy levels cross the graphene electronic states. Here, we observe avoided crossings in the band structure of the hybrid material, cf. Fig. 1(c).

To address the Förster energy transfer, we furthermore calculate the molecular transition dipole moment 
$\mathbf{d}_{\mathbf{M}}=-e_{0} \int d \mathbf{r} \Phi_{M}^{l *}(\mathbf{r}) \mathbf{r} \Phi_{M}^{h}(\mathbf{r})$ with the molecular HOMO and LUMO wavefunctions $\Phi_{M}^{h}(\mathbf{r}), \Phi_{M}^{l}(\mathbf{r})$. Evaluating the wave functions obtained in our DFT calculations, we find for the dipole moment in the units of $\mathrm{e}_{0} \mathrm{~nm}$

$$
\mathbf{d}_{\mathrm{M}}=\left(d_{x}, d_{y}, d_{z}\right)=(-0.518,-0.043,-0.215)
$$

with the absolute value of $d_{M}=0.56 \mathrm{e}_{0} \mathrm{~nm}$. The dipole moment is calculated for the porphyrin molecule that has been fully geometrically relaxed in the presence of the graphene substrate. Following the approach of Swathi et al. [45], the Förster transfer rate reads

$\gamma_{F}=\frac{2 \pi}{\hbar} \sum_{\mathbf{k}_{\mathbf{i}}} \sum_{\mathbf{k}_{\mathbf{f}}}\left|\mathbf{d}_{\mathbf{M}} \cdot \nabla \varphi_{\mathbf{k}_{\mathbf{i}}, \mathbf{k}_{\mathbf{f}}}^{v c}\right|^{2} \delta\left(\varepsilon_{\mathbf{k}_{\mathbf{f}}}^{c}-\varepsilon_{\mathbf{k}_{\mathbf{i}}}^{v}-\Delta E_{M}\right)$,

expressing the interaction of the molecular transition dipole $\mathbf{d}_{\mathbf{M}}$ located in the electrostatic potential $\varphi_{\mathbf{k}_{\mathbf{i}}, \mathbf{k}_{\mathbf{f}}}^{v c}$ arising from the transition charge density of graphene $\rho_{\mathbf{k}_{\mathbf{i}}, \mathbf{k}_{\mathbf{f}}}^{v c}(\mathbf{r})=-e_{0} \Phi_{\mathbf{k}_{\mathbf{i}}}^{v *}(\mathbf{r}) \Phi_{\mathbf{k}_{\mathbf{f}}}^{c}(\mathbf{r})$. The sums in Eq. (2) are performed over all momentum-dependent initial and final states of graphene $\Phi_{\mathbf{k}_{\mathbf{i}}}^{v}(\mathbf{r}), \Phi_{\mathbf{k}_{\mathbf{f}}}^{c}(\mathbf{r})$ considering the energy conservation expressed by the appearing delta function that includes the energy of the involved graphene states in the conduction and the valence band $\varepsilon_{\mathbf{k}_{\mathbf{f}}}^{c}, \varepsilon_{\mathbf{k}_{\mathbf{i}}}^{v}$ and the energy $\Delta E_{M}$ of the excited molecular transition.

Assuming the nearest-neighbor tight-binding approximation for the graphene wave functions [47], the transition charge density of graphene can be explicitly calculated allowing an analytic expression for the Förster energy transfer rate [45]

$$
\begin{aligned}
\gamma_{F}(R) & =\int_{0}^{\frac{\Delta E_{M}}{\nu_{F}}} \frac{e_{0}^{2}}{128 \pi \hbar \epsilon_{0}^{2}}\left(d_{\|}^{2}+2 d_{\perp}^{2}\right) \frac{e^{-2 q R} q^{3}}{\sqrt{\Delta E_{M}^{2}-\nu_{F}^{2} q^{2}}} d q \\
& \approx \frac{3 e_{0}^{2}}{1024 E_{M} \pi \hbar \epsilon_{0}^{2}} \frac{d_{\|}^{2}+2 d_{\perp}^{2}}{R^{4}} \text { for } R>1 \mathrm{~nm}
\end{aligned}
$$

with the molecule-substrate distance $R$, the momentum transfer $q$, and the slope in the electronic band structure of graphene $\nu_{F}$. The Förster rate explicitly depends on the substrate-molecule distance $R$ and on the square of the parallel $d_{\|}$and the perpendicular component $d_{\perp}$ of the molecular transition dipole moment $\mathbf{d}_{\mathbf{M}}$. The investigated structure lies in the $\mathrm{x}-\mathrm{y}$ plane, i.e. $d_{\|}^{2}=0.27$ and $d_{\perp}^{2}=0.05 \mathrm{e}_{0}^{2}$ $\mathrm{nm}^{2}$ resulting from $\mathrm{Eq}(1)$. Since the geometrically relaxed porphyrin molecule does not lie flat on the graphene layer (the four phenyl rings are rotated out of the plane), there is also a dipole component perpendicular to the graphene layer. The orientation of the molecular dipole moment enters implicitly into the Förster rate in Eq. (3), since the components of the dipole moment have different weights.

Figure 2 illustrates the Förster rate as a function of the substrate-molecule distance $R$ for the porphyrinfunctionalized graphene. Provided the knowledge of the

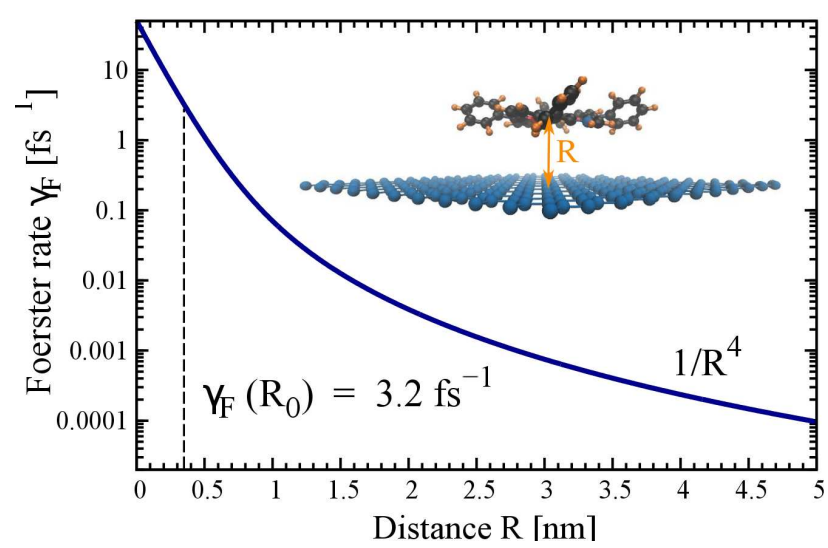

Figure 2 Distance dependence of the energy transfer between the graphene layer and the attached porphyrin molecule via Förster coupling. At the calculated optimal molecule-substrate distance $R_{0}=0.35 \mathrm{~nm}$, we obtain a Förster rate of $\gamma_{F}=3.2 \mathrm{fs}^{-1}$ suggesting a very strong energy transfer. For large distances, the Förster coupling exhibits a $R^{-4}$ dependence - as expected for spatially extended two-dimensional substrates $[45,24]$.

molecular transition dipole moment $d_{\|}, d_{\perp}$, the Förster rate can be easily extracted also for other graphene-based hybrid nanostructures. In the limit of large substratemolecule distances $R$, the Förster coupling exhibits a $R^{-4}$ dependence in contrast to the well-known $R^{-6}$ scaling for the Förster coupling in molecule-molecule complexes [40]. This can be traced back to the spatially extended two-dimensional graphene substrate [45] and is in good agreement with recent experimental observations [24]. For typical distances of $R=1-5 \mathrm{~nm}$ involving the presences of linker molecules that are sometimes necessary to realize the functionalization [32], we find Förster rates in the range of $\gamma_{F}=50-0.1 \mathrm{ps}^{-1}$. For small distances $R<1 \mathrm{~nm}, \gamma_{F}$ exhibits an exponential decay with increasing $R$. For the calculated minimal distance of $R_{0}=0.35 \mathrm{~nm}$ between the porphyrin molecule and the graphene substrate, we obtain a very efficient rate of $\gamma_{F}\left(R_{0}\right)=3.2 \mathrm{fs}^{-1}$. The large transition dipole moment of the adsorbed porphyrin molecule in combination with the very short substrate-molecule distance results in a strong Förster coupling between the molecule and the electrons within the graphene layer. Our results are in agreement with the experimentally observed strong energy transfer in porphyrin-functionalized carbon nanotubes, in particular with respect to the time-resolved transient absorption studies revealing that the energy transfer occurs on a femtosecond timescale [39].

In summary, we have investigated the Förster energy transfer in porphyrin-functionalized graphene. After having characterized the hybrid nanostructure within a study based on density functional theory, we calculate the energy transfer rate within the Fermi's golden rule. For the obtained molecule-substrate distance, we find an extremely efficient Förster energy transfer between the adsorbed porphyrin molecules and the graphene substrate. The pre- 
dicted values in the range of $f s^{-1}$ are in agreement with recent experimental results. Further studies are needed to calculate the Dexter energy transfer mechanism in these hybrid nanostructures.

We acknowledge financial support from the German Science Foundation for support within the collaborative research center 658. Furthermore, E.M. is thankful to the Einstein Foundation Berlin, O. T. H. to FWF (Project: J 3285-N20), and A.R acknowledges funding from the European Research Council (ERC-2010-AdG-267374), Spanish Grant (FIS2010-21282-C02-01), Grupos Consolidados UPV/EHU del Gobierno Vasco (IT578-13), and the EU project (280879-2 CRONOS CP-FP7).

\section{References}

[1] J. Kong, N. Franklin, C. Zhou, M. Chapline, S. Peng, K. Cho, and H. Dai, Science 287(5453), 622 (2000).

[2] R. Chen, Y. Zhang, D. Wang, and H. Dai, J. Am. Chem. Soc. 123(16), 3838 (2001).

[3] M. S. Strano, J. Am. Chem. Soc. 125(51), 16148-16153 (2003).

[4] R. Khairutdinov, M. Itkis, and R. Haddon, Nano Lett. 4(8), 1529 (2004).

[5] A. Hirsch and O. Vostrowsky245, 193 (2005).

[6] M. Burghard, Surf. Sci. Rep. 58(1-4), 1-109 (2005).

[7] D. Guldi, H. Taieb, G. Rahman, N. Tagmatarchis, and M. Prato, Advanced Materials 17, 871-875 (2005).

[8] X. Guo, L. Huang, S. O'Brien, P. Kim, and C. Nuckolls, J. Am. Chem. Soc. 127(43), 15045 (2005).

[9] J. M. Simmons, I. In, V.E. Campbell, T. J. Mark, F. Léonard, P. Gopalan, and M. A. Eriksson, Phys. Rev. Lett. 98(8), 086802 (2007).

[10] M. Del Valle, R. Gutirrez, C. Tejedor, and G. Cuniberti, Nature Nanotechnology 2(3), 176 (2007).

[11] G. Magadur, J. S. Lauret, V. Alain-Rizzo, C. Voisin, P. Roussignol, E. Deleporte, and J.A. Delaire, ChemPhysChem 9, 1250 (2008).

[12] J. P. Casey, S. M. Bachilo, and R. B. Weisman, J. Mater. Chem. 18, 1510-1516 (2008).

[13] R. S. Sundaram, C. Gomez-Navarro, K. Balasubramanian, M. Burghard, and K. Kern, Adv. Mater. 20, 3050 (2008).

[14] X. Zhou, T. Zifer, B. M. Wong, K. L. Krafcik, F. Leonard, and A. L. Vance, Nano Lett. 9(3), 1028 (2009).

[15] C. Ehli, C. Oelsner, D. M. Guldi, A. Mateo-Alonso, M. Prato, C. Schmidt, C. Backes, F. Hauke, and A. Hirsch, Nat. Chem. 1(3), 243-249 (2009).

[16] E. Del Canto, K. Flavin, M. Natali, T. Perova, and S. Giordani, Carbon 48(10), 2815 (2010).

[17] E. Malić, C. Weber, M. Richter, V. Atalla, T. Klamroth, P. Saalfrank, S. Reich, and A. Knorr, Phys. Rev. Lett. 106(9), 097401 (2011).

[18] A. M. Kolpak and J. C. Grossman, Nano Lett. 11, 3156 (2011).

[19] E. Malić, A. Setaro, P. Bluemmel, C. F. Sanz-Navarro, P. Ordejon, S. Reich, and A. Knorr, J. Phys. Condens. Matter 24, 394006 (2012).

[20] A. Setaro, P. Bluemmel, C. Maity, S. Hecht, and S. Reich, Adv. Funct. Mat. 22(11), 2425-2431 (2012).
[21] J. D. Correa and W. Orellana, Journal of Applied Physics 113(17), 174305 (2013).

[22] G. Berghäuser and E. Malić, Phys. Status Solidi B 250, 2678 (2013).

[23] F. Ernst, T. Heek, A. Setaro, R. Haag, and S. Reich, Appl. Phys. Lett. 102(23), 233105 (2013).

[24] L. Gaudreau, K. J. Tielrooij, G. E. Prawiroatmodjo, J. Osmond, F. J. G. de Abajo, and F. H. Koppens, Nano Lett. 13, 2030 (2013).

[25] G. Berghäuser and E. Malić, Carbon 69, 536 (2014).

[26] A. K. Geim and K.S. Novoselov, Nature Mat. 6, 183 (2007).

[27] P. Avouris, Z. Chen, and V. Perebeinos, Nature Nanotechnology 2(10), 605 (2007).

[28] F. Bonaccorso, Z. Sun, T. Hasan, and A. C. Ferrari, Nature Photonics 4(9), 611 (2010).

[29] T. Winzer, A. Knorr, and E. Malić, Nano Lett. 10(12), 4839 (2010).

[30] S. Winnerl, F. Göttfert, M. Mittendorff, H. Schneider, and M. H. et al., J. Phys. Condens. Matter 25.

[31] F. Wendler, A. Knorr, and E. Malić, Nature Commun. 5, 3703 (2014).

[32] F. Ernst, T. Heek, A. Setaro, R. Haag, and S. Reich, Adv. Funct. Mat. 22(18), 3921-3926 (2012).

[33] E. Kymakis and G. A. J. Amaratunga, Applied Physics Letters 80(1), 112-114 (2002).

[34] P. W. Barone, R. S. Parker, and M. S. Strano, Analytical Chemistry 77(23), 7556-7562 (2005).

[35] F. Lu, L. Gu, M. J. Meziani, X. Wang, P. G. Luo, L. M. Veca, L. Cao, and Y.P. Sun, Advanced Materials 21(2), 139-152 (2009).

[36] C. Roquelet, D. Garrot, J. S. Lauret, C. Voisin, V. AlainRizzo, P. Roussignol, J. A. Delaire, and E. Deleporte, Appl. Phys. Lett. 97, 141918 (2010).

[37] C. Roquelet, J. S. Lauret, , V. Alain-Rizzo, C. Voisin, and R. F. et al.

[38] C. Roquelet, F. Vialla, C. Diederichs, P. Roussignol, C. Delalande, E. Deleporte, J.S. Lauret, and C. Voisin, ACS Nano 6(10), 8796-8802 (2012).

[39] D. Garrot, B. Langlois, C. Roquelet, T. Michel, P. Roussignol, C. Delalande, E. Deleporte, J. Lauret, and C. Voisin, J. Phys. Chem. C 115, 23283-23292 (2011).

[40] T. Förster, Ann. Physik 437, 55 (1948).

[41] D. L. Dexter, J. Chem Phys. 21, 836 (1953).

[42] E. Malić, H. Appel, O. T. Hofmann, and A. Rubio, J. Phys. Chem. C 118, 9283 (2014).

[43] V. Blum, R. Gehrke, F. Hanke, P. Havu, V. Havu, X. Ren, K. Reuter, and M. Scheffler, Comput. Phys. Commun. 180, 2175 (2009).

[44] A. Tkatchenko and M. Scheffler, Phys. Rev. Lett. 102, 073005 (2009).

[45] R. S. Swathi and K. L. Sebastian, J. Chem. Sci. 121, 777 (2009).

[46] P. Perdew, K. Burke, and M. Ernzerhof, Phys. Rev. Lett. 77, 3865 (1996).

[47] E. Malić and A. Knorr, Graphene and Carbon Nanotubes: Ultrafast Optics and Relaxation Dynamics (WILEY-VCH, Berlin, 2003). 